J. PHYS IV FRANCE 7 (1997)

Colloque C3, Supplément au Journal de Physique III d'août 1997

\title{
Étude de la transition fragile-ductile d'un tungstène
}

\author{
J. Farre, M. Lamaison, A. Cosculluela* et J.-L. Lataillade** \\ Commisariat à l'Énergie Atomique, Centre de Valduc, B.P. 14, 21120 Is-sur-Tille, France \\ * Commisariat à l'Énergie Atomique, Centre du Ripault, B.P. 16, 37260 Monts, France \\ ** LAMEF/ENSAM, Esplanade des Arts et Métiers, 33405 Talence, France
}

\begin{abstract}
The aim of this study consists in analyzing the ductile-brittle transition temperature of a sintered tungsten. We lay emphasis on the appraisal of the influence of the triaxial stress state, of the strain rate and of the applied loading direction on the transition temperature. A simple modeling based on local competition between cleavage stress and theorical elastic yield stress is presented. It allows to appraise the transition temperature of a material under any loading condition.
\end{abstract}

Résumé: L'étude présentée a pour. but d'analyser la température de transition fragile ductile d'un tungstène fritté. Nous insistons sur l'évaluation de l'influence d'un champ triaxial de containtes, de la vitesse de déformation et de la direction de chargement sur la température de transition. Une modélisation simple basée sur la compétition locale entre la contrainte de clivage et la limite d'élasticité théorique est présentée. Elle permet d'évaluer la température de transition d'un matériau soumis à un chargement quelconque.

La plupart des matériaux polycristallins à structure cubique centrée est fragile à basse température. Aussi, le tungstène présente une transition de comportement pour des températures croissantes. En fait cette transition, souvent caractérisée par une température, dépend de nombreux paramètres liés, d'une part, à la microstructure (impuretés, taille de grains, texture ...) et, d'autre part, aux conditions de chargements (champ de contraintes, vitesse de déformation, mode de chargement...). A partir d'un seul tungstène nous allons étudier uniquement l'influence des conditions de chargement.

\section{MATERIAU D'ETUDE}

Le tungstène étudié est élaboré par frittage sous atmosphère d'hydrogène. Il est ensuite forgé à une température de $1000^{\circ} \mathrm{C}$. Des études des constantes élastiques du barreau (de diamètre $\Phi 30 \mathrm{~mm}$ ) suivant toutes les directions (axial, radial, orthoradial) ont montré que le tungstène est isotrope en rigidité et son module d'Young avoisine $400 \mathrm{GPa}(410 \mathrm{GPa}$ à $20{ }^{\circ} \mathrm{C} ; 380 \mathrm{GPa}$ à $\left.850{ }^{\circ} \mathrm{C}\right)$. Le matériau analysé présente une population de grains orientés préférentiellement (figure 1). Cette texture est apparue lors du forgeage à une température inférieure à la température de recristallisation $1420^{\circ} C[1,2]$. Les grains du matériau d'étude sont orientés suivant l'axe $<101>$, parallèlement à l'axe du barreau. L'ensemble des essais sera effectué dans la direction de l'axe d'orientation préférentielle.

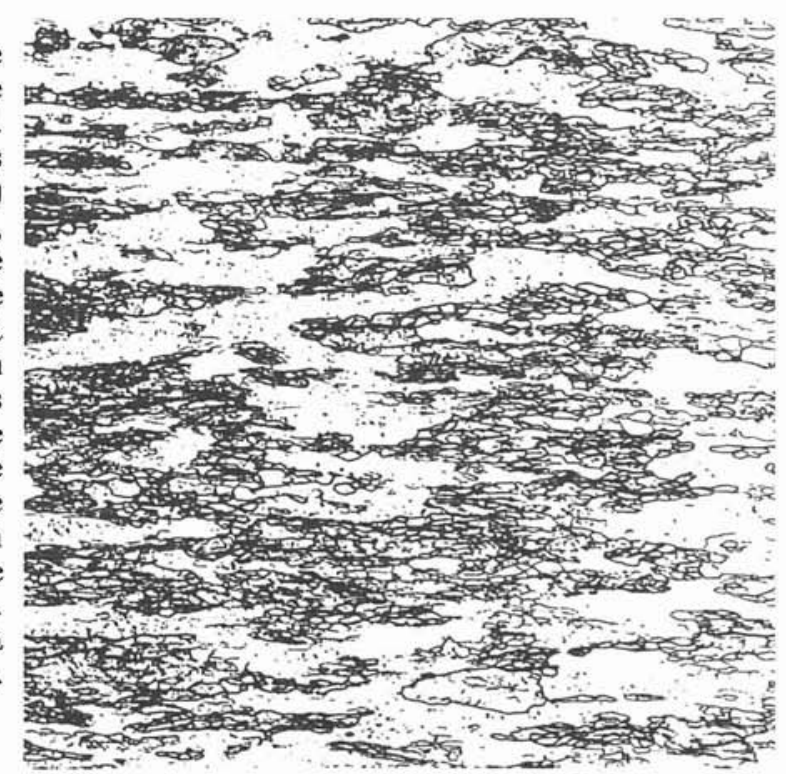

Figure 1 - Coupe suivant l'axe du barreau - $50 \mu \mathrm{m}$ 


\section{PRESENTATION DE LA TEMPERATURE DE TRANSITION FRAGILE-DUCTILE DU TUNGSTENE}

Schématiquement (figure 2), la température de transition fragile-ductile d'un matériau métallique peut être définie par l'égalité entre la limite d'élasticité et la contrainte de clivage du matériau. Pun une température inférieure à $T_{1}$ la rupture est fragile, le matériau rompt avant de plastifier. Entre $T_{1}$ et $T_{2}$, le matériau plastifie mais la rupture se fait par clivage. Au dessus de $T_{2}$, le matériau plastifie et la rupture s'effectue par cisaillement ductile. La température de transition, comprise entre $T_{1}$ et $T_{2}$, peut cependant être définie et identifiée par différentes techniques: mesures de déformation ou d'énergie à rupture, observations des faciès de rupture...[3].

En outre, il existe certains résultats sur la température de transition fragile ductile de tungstènes $[4,5,6]$ mais aucune étude ne s'est penchée sur une analyse comparative des différentes conditions de chargement (vitesse de déformation, entailles, mode de chargement).

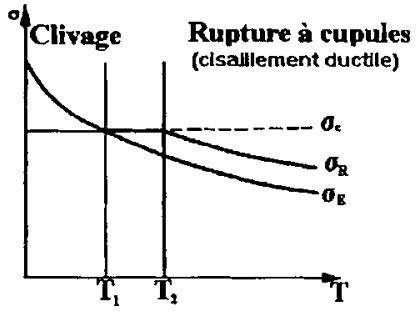

Figure 2 - Diagramme de Davidenko [7]

\section{PROCEDURE EXPERIMENTALE}

Ainsi, plusieurs moyens expérimentaux sont mis en oeuvre de manière à évaluer le rôle du champ de contraintes, de la vitesse de déformation et du mode de chargement.

En premier lieu, différents champs de contraintes peuvent être obtenus avec des éprouvettes similaires, les unes entaillées en $V$ et les autres en $Y$ (figure 3 ). Des essais de flexion trois points à une vitesse de déformation de l'ordre de $100 \mathrm{~s}^{-1}$ ont été effectués sur ces deux types d'éprouvettes.

Les essais réalisés sont respectivement de type Charpy avec four attenant et de type Hopkinson avec four in situ. Les chauffages sont réalisés sous balayage d'argon. La température est relevée au niveau de l' échantillon (caméra infra-rouge et thermocouple tungstène-rhénium, respectivement)

En second lieu, l'influence de la vitesse de déformation peut être étudiée à partir de la comparaison d'essais de flexion trois points statique $\left(10^{-3} s^{-1}\right.$, vitesse déterminée par simulation numérique sur CASTEM) et dynamique (environ $10^{2} s^{-1}$ ) sur les éprouvettes entaillées en Y. 'ne machine servo hydraulique Schenck équipée d'une cellule de force de $5 \mathrm{kN}$ est utilisée pour la flexion trois points quasi statique. Des thermocouples sont placés en contact avec l'éprouvette.

La détermination de la température fragile-ductile se fera par la mesure de l'énergie surfacique de rupture $\left(\frac{E_{\text {abserbéce }}}{S_{\text {fissuration }}}\right)$ pour différentes températures. La courbe de transition sera modélisée par

$$
E_{t}(T)=E_{1}+\frac{E_{2}-E_{1}}{2} \cdot\left[1+t h\left(\frac{T-T_{D}}{d T}\right)\right]
$$

où $E_{t}$ est l'énergie surfacique absorbée, $E_{1}$ l'énergie au palier fragile, $E_{2}$ l'énergie au palier ductile t: $T_{D}$ la température de transition. Aussi $d T$ caractérise la largeur de transition. Pour des problèmes de poinçonnement du système expérimental sous sollicitations quasi statiques nous mesurerons l'énergie absorbée par le matériau pour un taux de déformation plastique fixé.

En dernier lieu, pour comparer l'influence de la direction de chargement, des essais de compression sur des éprouvettes cylindriques $(\Phi: 6 \mathrm{~mm}, \mathrm{~h}: 6 \mathrm{~mm})$ et des essais de traction sur des éprouvettes à section carrée $\left(3 \times 5 \times 25 \mathrm{~mm}^{3}\right)$ ont été effectués à $10^{-3} \mathrm{~s}^{-1}$ à l'ambiante sur une machiné éléctromécanique de capacité $100 \mathrm{kN}$.

\section{RESULTATS EXPERIMENTAUX}

Comme nous le verrons dans le cinquième chapitre, le champ de contraintes en fond d'entaille eit différent suivant l'angle d'ouverture de la fissure. La température de transition est influencée par ce champ de contrainte (figure 4). Plus l'angle d'ouverture est faible plus la température est importanle A $100 \mathrm{~s}^{-1}$ les températures fragile-ductile des éprouvet tes cntaillóes en $\mathrm{V}$ et en Ý sont respectivm égales à $610^{\circ} \mathrm{C}+1-30^{\circ} \mathrm{C}$ et $795^{\circ} \mathrm{C}+1-40^{\circ} \mathrm{C}$. 
Aussi une augmentation de la vitesse de déformation se traduit par une augmentation de la température de transition (figure 5). Les éprouvettes entaillées en $\mathrm{Y}$ ont une température de transition de $795^{\circ} \mathrm{C}+/-40^{\circ} \mathrm{C}$ à $100 \mathrm{~s}^{-1}$ et de $350^{\circ} \mathrm{C}+/-50^{\circ} \mathrm{C}$ à $10^{-3} \mathrm{~s}^{-1}$.

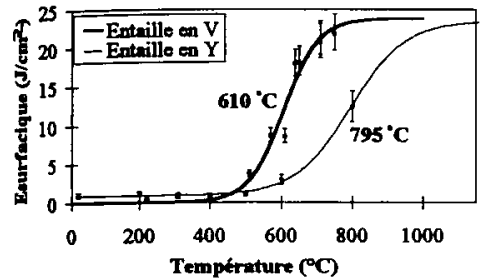

Figure 4 - Influence de la forme de l'entaille

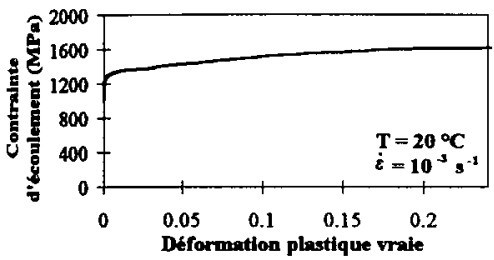

Figure 6 - Compression du tungstène

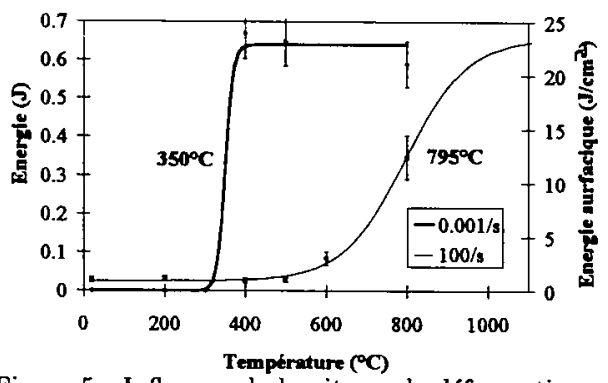

Figure 5 - Influence de la vitesse de déformation

En outre, à une même vitesse de déformation $\left(10^{-3} s^{-1}\right)$ et à une même température $\left(20^{\circ} \mathrm{C}\right)$, la rupture du matériau peut être soit fragile soit ductile suivant le mode de chargement. En traction le matériau a un comportement élastique jusqu'à rupture. Celle-ci est de type fragile et a lieu pour $\sigma=1000 \mathrm{MPa}[970$ et $1000 \mathrm{MPa}$. En compression, les courbes $\sigma-\epsilon$ (cinq essais reproductibles) traduisent un comportement plastique du tungstène (figure 6). La limite d'élasticité en compression est de $1250 \mathrm{MPa}$.

\section{ANALYSE DE LA TRANSITION}

A présent nous proposons une modélisation simple du phénomène de transition basée sur la compétition locale entre la limite d'élasticité et la contrainte de clivage. L'évolution du module d'Young (proportionnelle à cette dernière[8]) en fonction de la température étant faible nous posons une contrainte de clivage indépendante de la température. La valeur de celle-ci, $\sigma_{c}$, est donc notre première inconnue. La limite d'élasticité théorique est modélisée par une loi d'écoulement de type Steinberg-Lund [9](cf. formulation adoptée ci-dessous). Une optimisation des paramètres a donc été réalisée à partir d'essais de compression à différentes températures $\left(20^{\circ} \mathrm{C}\right.$ et $\left.600^{\circ} \mathrm{C}\right)$ et à différentes vitesses de déformation $\left(10^{-3} s^{-1}\right.$ et $\left.10^{3} s^{-1}\right)$.

$$
\sigma=\left[Y_{p} \cdot\left(1-\sqrt{\frac{k T}{2 U_{k}} \cdot \ln \left(\frac{C_{1}}{\dot{\epsilon}}\right)}+Y_{A} \cdot(1+\beta \epsilon)^{n}\right] \cdot[1-B(T-300)]\right.
$$

$Y_{p}=2200 \mathrm{MPa} ; U_{k}=0,77 \mathrm{eV} ; C_{1}=6,9.10^{5} \mathrm{~s}^{-1} ; Y_{A}=440 \mathrm{MPa} ; \beta=20 ; n=0,19 ; B=1,6.10^{-4} K^{-1}$.

Arbitrairement nous définissons la contrainte d'écoulement à $5 \%$ de déformation, et non la limite d'élasticité, comme valeur de contrainte intervenant dans la compétition locale avec la contrainte de clivage. Elle nous est suggérée par des définitions classiques de la température fragile-ductile [3].

\subsection{Effet du champ de contraintes - Effet des entailles}

La présence d'entaille influence la température de transition fragile ductile. Précisons que pour cette modélisation nous nous plaçons en hypothèse de déformations planes et nous utilisons comme critère d'équivalence celui de Tresca. 
En dessous d'une certaine température la limite d'élasticité est supérieure à $\sigma_{c}$ et le matériau est purement fragile (diagramme de Davidenko). Au dessus de cette température, en fond d'entaille, va apparaitre une zone plastique (figure 7) et le champ de contraintes est défini dans cette zone par les équations ci-dessous.

$$
\begin{gathered}
\sigma_{\theta \theta}=R_{e}\left[1+\ln \left(1+\frac{x}{R}\right)\right] \quad \sigma_{r r}=R_{e} \cdot \ln \left(1+\frac{x}{R}\right) \\
\sigma_{z z}=\frac{\sigma_{\theta \theta}+\sigma_{r r}}{2} ; \quad R_{e}: \text { limite d'élasticité en traction [8] }
\end{gathered}
$$

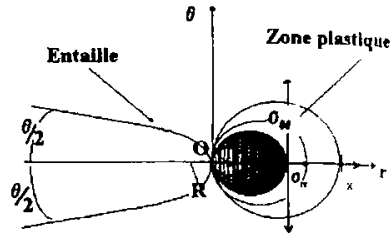

Figure 7 - Champ de contraintes en fond d'entailles

Si la contrainte maximale $\sigma_{\theta \theta \max }$, localisée à la frontière de la zone plastifiée, atteint la contrainte. de clivage $\sigma_{c}$, le matériau rompt par clivage mais présente une petite zone plastifiée en fond d'entaille. Si cette contrainte n'atteint pas $\sigma_{c}$ la zone plastifiée s'étend jusqu'à une taille maximale telle que la distance entaille-limite de la zone plastique soit égale à $x=R \cdot\left[\exp \left(\frac{\pi}{2}-\frac{\theta}{2}-1\right)\right]$ d'où :

$$
\sigma_{\theta \theta \max }=R_{e} \cdot\left(1+\frac{\pi}{2}-\frac{\theta}{2}\right)
$$

Ensuite c'est l'écrouissage $\left(R_{e}=R_{e}(\epsilon)\right)$ qui provoque une augmentation de la contrainte maximale tant que la contrainte de clivage n'est pas atteinte. Mais lorsque $\sigma_{\theta \theta \max }$ est suffisamment faible (diminution avec l'élévation de la température), la contrainte de clivage n'est jamais atteinte et la rupture est totalement ductile. Il existe donc deux transitions, la transition de ductilité nulle et la transition semi-fragile-ductile. Cette dernière est donc directement reliée à la limite d'élasticité par:

$$
\sigma_{c}\left(T_{D}\right)=R_{e}\left(T_{D}\right) \cdot\left(1+\frac{\pi}{2}-\frac{\theta}{2}\right)
$$

Pour les éprouvettes entaillées en $\mathrm{V}$, l'angle d'ouverture $\theta$ est égale à $\frac{\pi}{4}$, donc $\sigma_{c}=2,18 R_{e}$, alors que pour les échantillons entaillés en $\mathrm{Y}$, $\theta=0$, d'où $\sigma_{c}=2,57 R_{e}$. Nous avons accès à l'évolution de la contrainte d'écoulement à $5 \%$ de déformation via le modèle de la loi d'écoulement (figure 8). Ainsi à partir de la valeur de la température de transition fragile-ductile $\left(610^{\circ} \mathrm{C}+/-30^{\circ} \mathrm{C}\right)$ évaluée lors des essais sur des éprouvettes entaillées en $V$ (vitesse de déformation de l'ordre de $100 \mathrm{~s}^{-1}$ ), nous déterminons la contrainte de clivage: $\sigma_{c}=2,18 . R_{e}(5 \%)$ (figure Figure 8 - Influence du champ de contraintes 8) soit $\sigma_{\mathrm{c}}=2600 \mathrm{MPa}[2540 ; 2660]$.

Nous pouvons aussi en déduire la température de transition semi-fragile-ductile pour l'entaillea $\theta=0$ à partir de la courbe $2.57 \operatorname{Re}=\mathrm{f}(\mathrm{T})($ figure 8$)$ : elle est de $820^{\circ} \mathrm{C}+/-30^{\circ} \mathrm{C}$.

L'analyse présentée ne tient pas compte d'une éventuelle élévation de température en fond de fissure due à une plastification locale. A de telles vitesses de déformation les essais peuvent ètre considérés adiabatiques. Cette élévation conduirait en fait à des valeurs de transition plus élevées et à une valeur de contrainte de clivage plus faible. Cette élévation de température, difficilement quantifiable, est cependant similaire pour les deux types d'éprouvettes. Ainsi, à partir d'essais réalisés sur des éprouvettes entaillées en $V$ et d'un modèle simple nous estimons la température de transition d'éprouvettes entaillées en Y. La comparaison avec la valeur expérimentale valide la modélisation.

\subsection{Influence de la vitesse de déformation}

Pour des éprouvettes identiques (ici entaillées en Y) quelle que soit la vitesse de déformation la température de transition $T_{D}$ sera définie par $\sigma_{c}\left(T_{D}\right)=\alpha \cdot R_{e}\left(T_{D}, \dot{\epsilon}\right)$ (ici $\alpha=2.57$ ).

Ainsi, à partir de $\sigma_{c}=2600 \mathrm{MPa}$, nous pouvons déterminer analytiquement la valeur de la température de transition en flexion trois point,s pour les éprouvettes entaillées on $Y$ sollicitt: à une vitesse de déformation de $10^{-3} \mathrm{~s}^{-1}$. Elle est de $225+/-15^{\circ} \mathrm{C}$. Or expérimentalencul 
susdite température avait été mesurée à $350+/-50^{\circ} \mathrm{C}$. Les différences observées peuvent s'expliquer par plusieurs points: une optimisation imparfaite du modèle, des valeurs de vitesse de déformation approximatives pour les essais de flexion dynamique, l'échauffement local du matériau non pris en compte pour la détermination de $\sigma_{c}$.

Comme les élévations de températures pouvaient être considérées identiques pour les études précedentes le modèle validé au paragraphe 5.1. n'est pas remis en cause. Cependant afin de tenir compte de l'élévation de température nous redéterminons $\sigma_{c}$ à partir des essais quasi statiques de flexion $\left(\mathrm{T}_{D}=350^{\circ} \mathrm{C}\right)$. Ces essais, suffisamment lents, sont isothermes et ne présentent aucune élévation de température. Alors, par une nouvelle comparaison des valeurs modélisée et expérimentale $\left(T_{D}=795^{\circ} \mathrm{C}\right)$ pour les essais dynamiques sur les éprouvettes entaillées en $\mathrm{Y}$ nous estimons cette élévation $\Delta T$ (figure 9 ).

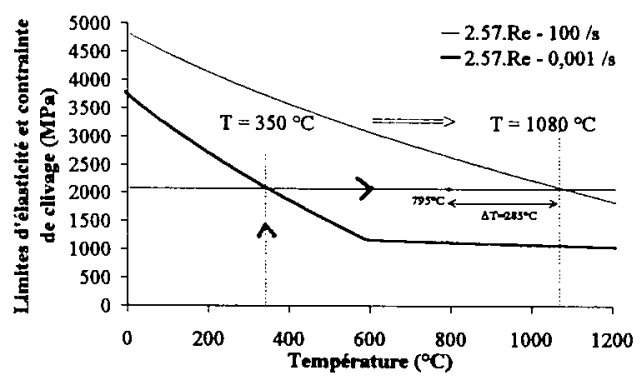

Figure 9 - Influence de la vitesse de déformation

La contrainte de clivage macroscopique est de $2080 \mathrm{MPa}[1880-2240]$. L'élévation locale de température lors des essais dynamiques proches de la transition est de $\Delta T=1080-795=285^{\circ} \mathrm{C}$ $\left(T_{D}=1080^{\circ} C[1000 ; 1190]\right)$. Une telle élévation de température correspondrait à $90 \%$ de l'énergie plastique créée jusqu'à $20 \%$ de déformation:

$$
\Delta T=\frac{0,9}{\rho C_{p}} \cdot \int\left(\sigma_{\theta \theta} \cdot d \epsilon_{\theta \theta}+\sigma_{r r} \cdot d \epsilon_{r r}\right)
$$

( $\rho$ : masse volumique, $C_{p}$ : chaleur spécifique).

Ainsi nous pouvons présenter un deuxième paramètre du modèle: $\Delta T$, une éventuelle élévation locale de la température (pour des essais adiabatiques) correspondant à une plastification jusqu'à $20 \%$ de déformation.

\section{Validation}

Nous pouvons valider le modèle à partir des essais sur éprouvettes entaillées en V. Pour $\sigma_{\mathrm{c}}=2080 \mathrm{MPa}$ la température modélisée est de $\mathrm{T}=890^{\circ} \mathrm{C}$. La température avant plastification locale est de $\mathrm{T}-\Delta T=605^{\circ} \mathrm{C}$. Expérimentalement la valeur était de $610^{\circ} \mathrm{C}$.

\subsection{Influence du mode de chargement}

Le modèle présenté retranscrit bien les influences des entailles et de la vitesse de déformation sur la température fragile-ductile du tungstène étudié. Or nous avons mis en évidence une différence du comportement à rupture sous sollicitations uniaxiales: il est fragile en traction et ductile en compression (à $\left.10^{-3} s^{-1}\right)$.

Nous présentons donc ici une interprétation simple toujours basée sur la compétition locale de $\sigma_{c}$ et $R_{e}$. Dans les deux cas la plasticité apparaîtra lorsque la contrainte $|\sigma|$ atteindra $R_{e}$. En traction la contrainte maximale pouvant créer une rupture par clivage est $\sigma$, soit $\sigma_{c}=\sigma$, alors qu'en compression la contrainte $-\sigma$ ne peut évidemment pas créer une telle rupture. La rupture en compression aura lieu en cisaillement, soit pour $\sigma_{c}=\frac{\sigma}{2}$. In fine, les températures de transition sont définies par deux égalités différentes:

en traction: $\sigma_{c}\left(T_{D}\right)=R_{e}$;

$$
\text { en compression: } \sigma_{c}\left(T_{D}\right)=\frac{R_{c}}{2} \text {. }
$$

Il en résulte une température de transition plus basse en compression qu'en traction. Un matériau sans défaut aurait en traction une contrainte à rupture égale à la contrainte de clivage. $0_{r}$, expérimentalement cette valeur est de moitié. Il existe apparemment des concentrations locales de contraintes qui induisent des champs triaxiaux. Or nous savons que l'introduction d'une entaille en $V$ abaisse la température de transition[10], donc le facteur $\alpha$ est inférieure à 2,18. Si localement nous considérons arbitrairement un défaut hexagonal, l'angle d'ouverture de la fissure est de $\frac{\pi}{3}$, soit en traction, à $T_{D}, \sigma_{c}=2 R_{e}$ et la contrainte à l'infini à rupture du matériau fragile est de $\frac{\sigma_{c}}{2}$. De la même manière, en compression, à $T_{D}, \sigma_{c}=R_{e}$. 
A $10^{-3} \mathrm{~s}^{-1}$, la température modélisée en traction est de $150^{\circ} \mathrm{C}$ et de $60 \mathrm{~K}$ en compression. ( $\mathrm{e}$ qui confirme le fait que la rupture est fragile en traction et ductile en compression.

\section{Critique}

Nous avons basé notre travail sur la compétition locale de la contrainte de clivage et la linitr: d'écoulement à $5 \%$. Or de par les valeurs d'élévation de température il aurait été plus licite d'utiliser la limite d'écoulement à $20 \%$. Cependant l'erreur faite sur la contrainte de clivage serait inférieure à $100 \mathrm{MPa}$. Nous confirmons la validité de l'étude et proposons une contrainte de clivage d'onviron $2100 \mathrm{MPa}$. Nous pouvons émettre une critique quant à la valeur incertaine du facteur " 2 " utilisé sous sollicitations uniaxiales. Une telle hypothèse entraînerait une contrainte à rupture (à l'infini). en traction, indépendante de la vitesse de déformation et égale à $\sigma_{r}=\frac{\sigma_{c}(\text { locale })}{2}=1040 \mathrm{MPa}[940,1120]$. Les valeurs des contraintes à rupture obtenues en traction semblent valider une telle hypothipus. N'oublions pas de signaler qu'il peut exister des incertitudes dues à la modélisation de la linite d'élasticité en compression appliquée en traction, sachant que le matériau est texturé.

\section{CONCLUSION}

A partir d'un modèle simple basé sur la compétition locale entre la contrainte de clivage et les linites d'écoulement théoriques et de deux séries d'essais de flexion trois points sur des éprouvettes coltailips: en $\mathrm{Y}$ quasi statiques et dynamiques, nous avons pu caracteriser macroscopiquement la température de transition fragile-ductile.

Deux types d'essais de validation ont été utilisés: des essais sur éprouvettes entaillées 'n l: valident la determination du champ de contrainte en fond d'entaille, des essais de traction sur des éprouvettes lisses valident la valeur de la contrainte de clivage obtenue.

Ici la contrainte de clivage du tungstène est d'environ $2100 \mathrm{MPa}$.

Un tel modèle peut permettre de déterminer par simulation la température fragile-ductile d'un matériau soumis à un chargement quelconque.

\section{Remerciements}

Les auteurs remercient G. Naulin (CEA Valduc) et A. Matagne (CEA Bruyères-le-Châtel) ainsi que le LAMEF-ENSAM (Bordeaux) pour leur aide.

\section{Références}

[1] Quarrell A.G.: Niobium, tantalum, molybdenum and tungsten, Department of Metallurgy University of Sheffield, 1991.

[2] Belhadjhamida A. et German R. : "Tungsten and tungsten alloys by powder metallurgy - A status review", Tungsten and Tungsten Alloys-recent Advances 120th Annual Meeting of Minerals. Metals and Materials society, 1991, New Orleans (US) February, pp3-19.

[3] Lassila D.H. et Connors A.: "Fracture behavior of warm forged and CVD tungsten", Tungste! and Tungsten Alloy-recent advances 120th Annual Meeting of Minerals, Metals and Materials society, New Orleans (US) February 1991, pp 79-85.

[4] Seigle L.L. et Dickinson C.D. : "Effect of mechanical and structural variables on the ductile-brittle transition in refractory metals", Refractory Metals and Alloys, 1962 pp 64-117.

[5] Zurek A.K. et GrayIII G.T.: "Dynamic strengh and strain rate effects on fracture behavior of tungsten and tungsten alloys", Journal de Physique IV, Colloque C3 suppl au Journal de Physiqu III, 1991. Vol 1 pp C3-631-C3-637.

[6] Chen S.R. et GrayIII G.T. : "Constitutive behavior of tungsten and tantalum : experiments and modeling", Tungsten and refractory metals 1994, pp 489-498.

[7] Francois D., Pineau A., Zaoui A.: Comportement mécanique des matériaux, Edition HER.1lls 1993, vol. 2

[8] Miannay D.: Mécanique de la rupture, les Editions de Physique, 1995

[9] Steinberg D.J. et Lund C.M. : "A constitutive model for strain rates from $10^{-4}$ to $10^{6} \mathrm{~s}^{-1 "}$.J. Appl. Phys. 65 (4), 15 February 1989, pp1528-1533

[10] Tetelman A.S. et Mc Evily A.J.: Fracture of structural materials, Editor John Wiley \& Son: 1967 